\title{
CDISC SEND Consciousness State Terminology
}

National Cancer Institute

\section{Source}

National Cancer Institute. CDISC SEND Consciousness State Terminology. NCI

Thesaurus. Code C90018.

The terminology that includes concepts relevant to the Clinical Data Interchange Standards Consortium (CDISC) Standard for the Exchange of Non-clinical Data (SEND) consciousness state. 